\title{
LE COURRIER DES LECTEURS, LIEU DE DÉBAT SUR LES MOUVEMENTS SOCIAUX QUI TOUCHENT LA BELGIQUE
}

\author{
Marie Widart ${ }^{1}$
}

\section{Introduction}

Depuis quelques années, les recherches en sciences de la communication se sont penchées sur le pôle longtemps ignoré des récepteurs. À ce titre, les rubriques du courrier des lecteurs, publiées par certains quotidiens et périodiques, offrent un accès privilégié à l'opinion des lecteurs et aux interactions établies entre eux et leur journal. Nous avons choisi, dans le présent article, d'analyser ces courriers à propos d'événements précis que sont les mouvements sociaux. Car les grèves, les manifestations, les arrêts de travail et les protestations en tout genre ne manquent pas de susciter de nombreuses réactions dans la population et, par ricochet, dans les rubriques du courrier des lecteurs. En effet, que l'on adhère aux revendications des protestataires ou que l'on condamne leur grève, la question laisse rarement indifférent. Concerné ou non, chacun en subit souvent peu ou prou les conséquences : retards dus aux débrayages à

1 Chercheur FNRS (Fonds National de la Recherche Scientifique) au Département de communication de l'Université catholique de Louvain.

Recherches en communication, $\mathrm{n}^{\circ} 21$ (2004). 
la SNCB, déviations et ennuis de circulation à cause d'une manifestation, enfants à "caser" quand les enseignants partent en grève... Tous ces mouvements, touchant au départ une catégorie "cible" de la population, finissent bien souvent par atteindre tout un chacun. C'est pourquoi, il nous a semblé intéressant d'étudier le courrier des lecteurs au travers de ces mouvements sociaux, riches en réactions.

La rubrique du courrier des lecteurs, espace laissé à la "parole usagère" au sein du journal, permet un croisement entre l'expression populaire et la sphère médiatique. Elle est l'interface, le relais médiatique du discours populaire et, à ce titre, un accès privilégié à l'opinion des lecteurs. Toutefois, si cette source présente une incontestable richesse, elle n'en connaît pas moins des limites. Ainsi, le courrier des lecteurs ne donne à lire que ceux qui veulent réagir et ne représente dès lors pas la totalité de la population. Nombreux sont ceux qui ont des opinions et des convictions mais ne les expriment pas. Par ailleurs, les journaux effectuent un tri parmi tout le courrier reçu et ne reproduisent pas toujours l'entièreté du texte ${ }^{1}$. Pourtant, malgré ses faiblesses, la rubrique du courrier des lecteurs demeure un témoin privilégié du point de vue du "récepteur". Car ces prises de parole, certes "contrôlées", nous font pénétrer dans l'univers des lecteurs.

Pour notre étude, nous avons sélectionné sept événements survenus en Belgique après la Deuxième Guerre mondiale. Nous les avons choisis selon deux critères: d'une part, une importance suffisante des faits pour avoir marqué les esprits et, d'autre part, d'un événement à l'autre, une occurrence assez régulière pour percevoir avec un peu de finesse les évolutions ou les permanences. Ainsi avons-nous retenu les événements suivants :

\footnotetext{
' En 1986 par exemple, à l'occasion des raids américains sur Tripoli, La Dernière Heure, envahie par les courriers sur la question, fait état, dans un "Avis aux lecteurs", du tri qu'elle opère. "Il nous reste, dans les réserves de cette rubrique, de très nombreuses lettres relatives au raid américain sur Tripoli et au danger que constitue l'attitude des intégristes musulmans. Malheureusement, le temps passe et les images que vous avez vues à la télévision et que vous commentez datent, à présent de plus d'un mois. Depuis, d'autres événements ont justifié vos réactions et, le courrier s'accumulant, nous avons décidé de ne plus tenir compte des réactions à ce raid, d'autant que vos arguments, intéressants et compétents, rejoignent la plupart de ceux que nous avons déjà exposés. Quant à savoir pourquoi nous avons retenu certaines lettres plutôt que d'autres, le hasard du dépouillement en a, seul, été responsable." Voir La Dernière Heure, le 21/05/1986, n 141, p. 9.
} 
- La guerre scolaire (1955): moment du conflit entre la majorité socialiste-libérale et l'enseignement libre à propos de la gestion de l'école, suite à la promulgation de la Loi Gilson ;

- Les grèves contre la loi unique (1960-1961): période de manifestations au cours de l'hiver 60 , particulièrement dans le sud du pays, en réaction aux mesures d'austérité prises par le gouvernement ;

- La grève des médecins (1964) : mouvement de grève des soins décidé par la Fédération nationale des Chambres syndicales de médecins suite au vote de la Loi Leburton destinée à conventionner le corps médical ;

- La manifestation des agriculteurs à Bruxelles (1971): considérée comme la première manifestation européenne, le 24 mars. Fut très violente et fit de nombreux dégâts dans la ville ;

— La manifestation des sidérurgistes à Bruxelles (1982) : suite aux problèmes de Cockerill-Sambre, descente à Bruxelles le 16 mars des sidérurgistes de Liège et de Charleroi : 179 policiers et cent manifestants seront blessés ;

- Les grèves dans les services publics (1986) : en mai, la grève affecte l'ensemble des services publics contre les mesures d'assainissement prises par le gouvernement dans le cadre des pouvoirs spéciaux ;

- La grève des enseignants (1990) face aux restrictions voulues par le gouvernement de la Communauté française.

Quelle place les divers journaux accordent-ils à l'expression de leurs lecteurs à propos de ces événements ? Qui sont ceux qui prennent la parole? Quel regard les lecteurs-écrivants portent-ils sur les grèves ? Quelles questions abordent-ils ? Comment interagissentils avec leur journal ? Quelles sont les grandes évolutions ou, au contraire, les permanences qui traversent les courriers en 35 ans ? Voilà autant de questions auxquelles le présent article tentera de répondre.

\section{Présentation du corpus}

Nous avons dépouillé des quotidiens nationaux et régionaux ainsi que quelques hebdomadaires nationaux. Pour l'ensemble des événements, nous avons trouvé 572 courriers dont seulement 14 dans des journaux régionaux (contre 558 dans la presse nationale). Ce 
déséquilibre montre bien que les rubriques de courrier des lecteurs restent l'apanage des quotidiens et hebdomadaires nationaux. Ainsi trouvons-nous, tous événements confondus, 218 courriers dans $L e$ Soir, 130 dans Pourquoi Pas ?, 76 dans La Dernière Heure, 72 dans La Libre Belgique, 29 dans Le Vif l'Express, 16 dans L'Écho de la Bourse, 9 dans Le Peuple, 7 dans La Cité, 6 dans La Nouvelle Gazette, 6 dans La Province et seulement 1 dans Le Soir Illustré, Vers l'Avenir et Le Courrier de l'Escaut. Aucun courrier n'est publié dans La Meuse (si ce n'est du courrier du cœur, abondant celui-là), Le Rappel, Le Courrier, Le Jour, La Nation Belge, Le Drapeau Rouge, La Wallonie, Le Patriote Illustré ni dans Spécial.

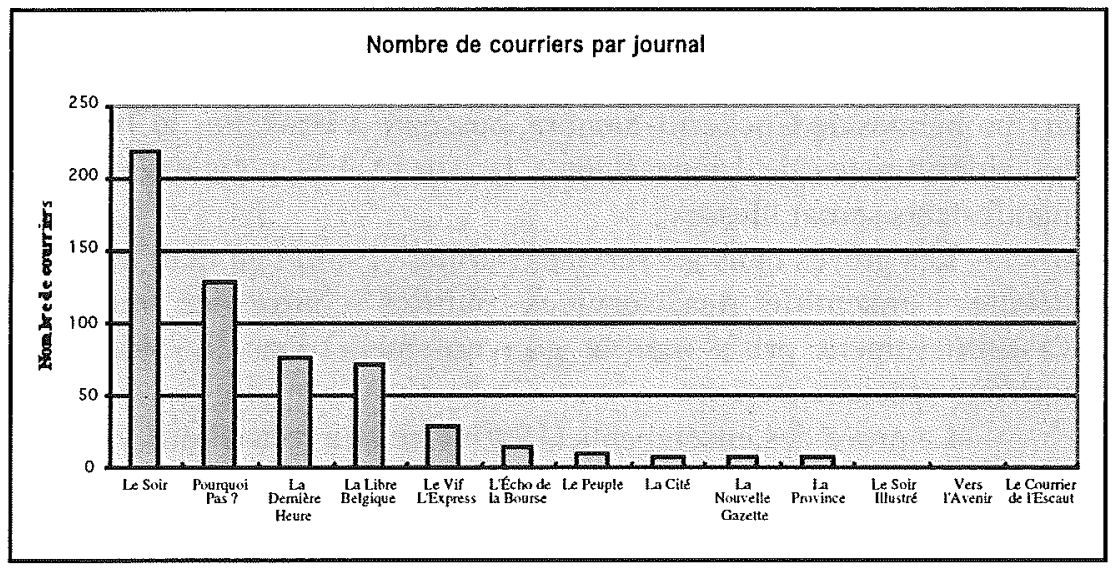

Le Pourquoi Pas? détient le quasi-monopole des courriers jusqu'à son déclin (au début des années 1970). Interlocuteur privilégié des lecteurs, il semble qu'il faille passer par lui pour avoir une place dans un journal. Ainsi, pour la guerre scolaire, tous les courriers émanent du Pourquoi Pas ?. Pour les grèves contre la loi unique, 27 des 31 courriers paraissent également dans cet hebdomadaire. Avec la grève des médecins, le Pourquoi Pas ? totalise encore 53 courriers sur les 58 publiés. Dans les années 1950 et 1960, le Pourquoi Pas ?, par sa politique rédactionnelle, paraît donc incontournable car il est à peu près le seul à ouvrir ses colonnes aux lecteurs. Quant au succès du 
journal Le Soir, il le doit à la grève des enseignants pour laquelle il publie près de 200 courriers ${ }^{1}$.

Par ailleurs, le nombre de courriers par événement se répartit comme suit : 31 pour la guerre scolaire (1955) et la loi unique (196061), 58 pour la grève des médecins (1964), 17 pour la manifestation des agriculteurs (1971), 83 pour les métallurgistes (1982), 52 pour les services publics (1986) et 300 pour les enseignants (1990).

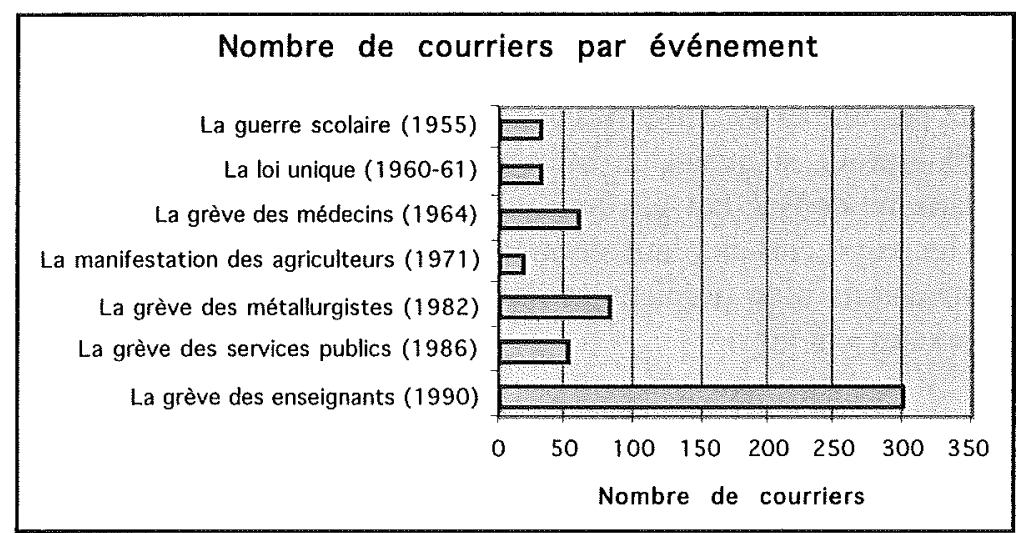

\section{La rubrique du courrier des lecteurs}

D'emblée, un constat : le nombre de courriers publiés varie fortement selon les événements. De 31 lettres les premières années, il passe par bien des fluctuations avant de culminer à 300 courriers en 1990. Comment expliquer ces évolutions ? Tout d'abord, la rubrique du courrier des lecteurs s'inscrit dans son époque et est le fruit de la politique rédactionnelle des journaux. Ainsi, le Pourquoi Pas? institue-t-il, dès sa création en 1910, une rubrique fournie, alors que d'autres journaux attendent les années septante pour établir une rubrique systématique. Il semble clair que, au lendemain de la première guerre, la presse s'intéresse peu au point de vue des lecteurs. Excepté le cas notoire du Pourquoi Pas ?, les autres périodiques se veulent essentiellement informationnels. Le seul contact avec le lecteur se fait à travers une rubrique de conseils sur des questions bien

1 L'attention accordée par Le Soir à ses lecteurs s'accroît nettement au début des années 1990 avec la création d'une rubrique beaucoup plus fournie qu'auparavant, intitulée tout simplement "le courrier des lecteurs". 
déterminées (réponses à des interrogations des lecteurs au sujet de leur pension, des allocations...). L'absence de rubrique du courrier des lecteurs traduit alors une certaine façon d'envisager le rôle de la presse et son rapport au lecteur. Celui-ci est considéré uniquement comme un récepteur auquel on livre des informations sans attente de retour. Quelques courriers occasionnels paraissent de-ci, de-là, au détour d'un article et à un rythme aléatoire. Pourtant, au vu du succès récolté par le Pourquoi Pas?, il semble que la demande soit bien présente $^{1}$. Les lecteurs sont prêts à écrire et à défendre leurs opinions par la plume. Mais le manque d'attention des autres journaux ne permet pas une expression massive de ces lecteurs-écrivants ${ }^{2}$.

Toutefois, si, jusqu'au milieu des années 1970, la plupart des journaux ne consacrent pas une rubrique régulière à l'expression de leurs lecteurs, certains leur donnent tout de même la parole, mais à des occasions bien précises et dans un cadre fixé. Le courrier des lecteurs devient ainsi

un lieu extrêmement codifié et balisé de la prise de parole du public. Il serait d'ailleurs plus correct de le définir comme un "lieu de don de parole" dans la mesure où il s'agit moins pour les lecteurs de prendre volontairement la parole que de se la

1 Le Pourquoi Pas? en vient même à expliquer au début de la rubrique "On nous écrit" du 10/04/1964 : "Impossible de publier l'avalanche de lettres commentant le conflit qui oppose le gouvernement aux médecins. Notre éditorial de cette semaine étant consacré, encore une fois, à cette importante question, nous nous réservons de reproduire dans notre prochain numéro un échantillonnage des commentaires que nos lecteurs nous auront fait parvenir d'ici là". Cette remarque montre bien que le désir d'écrire du public est présent. Et la semaine suivante, le résultat est là : 25 courriers sur les 27 publiés sont consacrés aux médecins. Et le Pourquoi Pas? d'introduire à nouveau sa rubrique : "Le conflit gouvernement-médecins a inspiré à nos lecteurs des commentaires tour à tour inquiets, désabusés, ironiques, énervés, voire passionnés. Ces avis, exprimés par centaines, vont de la condamnation formelle de la grève à l'approbation la plus totale des médecins révoltés, en passant par toutes sortes de nuances. Dans l'échantillonnage forcément limité que nous publions ci-après, nous nous sommes efforcé de respecter les proportions d'avis émis en faveur ou en défaveur des médecins". La semaine suivante, le 24/04/1964, le même scénario se reproduit et la rédaction du Pourquoi Pas ? écrit : "Encore une fois, nous prions nos lecteurs de bien vouloir nous excuser, mais il nous est matériellement impossible de publier les centaines de lettres qui nous ont été envoyées au sujet du conflit médecins-gouvernement".

2 Lors de la manifestation violente des agriculteurs à Bruxelles, par exemple, $L a$ Dernière Heure reconnaît avoir reçu un courrier abondant mais elle n'en publie pourtant aucun dans ses colonnes. 
voir attribuée (souvent partiellement) par les instances rédactionnelles des journaux ${ }^{1}$.

Les journaux incitent donc à l'occasion les lecteurs à s'exprimer sur un sujet de haute actualité, et notamment sur les mouvements sociaux importants. Le Peuple, par exemple, n'a pas de rubrique régulière de courriers des lecteurs mais, à l'occasion de la rentrée scolaire, publie un encart 'L'enseignement, ça nous concerne tous. Écrivez-nous. Nous publierons votre opinion"2.

Avec le milieu des années 1970 s'opère un net changement. Les rubriques du courrier des lecteurs se multiplient. Alors qu'auparavant la majorité des journaux, hormis le Pourquoi Pas?, ne publiait qu'occasionnellement des courriers, les rubriques régulières abondent dans la presse nationale. Ainsi, Le Vif l'Express, L'Écho de la Bourse, Le Soir, ou La Cité accordent désormais une place régulière à leurs lecteurs. Quant à La Libre Belgique et à La Dernière Heure, elles développent considérablement leur rubrique. De nombreux commentaires des rédactions attestent d'ailleurs de l'abondance du courrier reçu. En voici quelques extraits :

$\grave{A}$ en juger par le courrier qu'elle nous vaut, la manifestation récente des sidérurgistes à Bruxelles a causé un choc qui fait encore courir des ondes. L'exaspération née de la crise encourage évidemment l'expression de thèses opposées à l'extrême ${ }^{3}$.

Les mesures prises par le gouvernement pour redresser notre économie et assainir nos finances, et les commentaires que

1 G. DerÈze et S. MEYER, "Quand le public prend la parole. Analyse de courriers des lecteurs", dans B. GrevisSe (ss dir. de), L'affaire Dutroux et les médias. Une révolution blanche des journalistes?, Louvain-la-Neuve, Bruylant-Academia, 1999, p. 53.

2 Suit alors un texte: "La rentrée scolaire, c'est pour la semaine prochaine. Si vous avez un avis à émettre sur le statut des enseignants, sur le rôle des parents, sur l'organisation et la qualité de notre enseignement, sur la formation de notre jeunesse, etc... n'hésitez pas : écrivez-nous au "Journal et Indépendance - Le Peuple", 18, rue du Collège - 6000 Charleroi. Votre lettre devra nous parvenir le jeudi 6 septembre au plus tard. Merci de ne pas dépasser l'équivalent de 40 lignes manuscrites. Nous publierons le point de vue de nos lecteurs, votre point de vue, dans l'édition du "Journal et Indépendance - Le Peuple " du samedi 8 septembre ". Seuls trois courriers seront publiés à la date fixée. Le Peuple n'a donc pas de rubrique du courrier des lecteurs à proprement parler mais le journal donne la parole à ses lecteurs à une occasion bien précise : la rentrée.

3 Le Soir, le 06/05/82, n 104, p. 5. 
nous leur consacrons, nous valent un abondant courrier. Nous en publions ci-dessous quelques extraits qui nous paraissent représentatifs des principaux courants d'opinion. ${ }^{1}$.

La crise de l'enseignement et les grèves des enseignants nous valent un abondant courrier en sens divers. Il n'est évidemment pas possible de publier toutes les lettres que nous recevons. Du moins proposons-nous ci-dessous un florilège significatif ${ }^{2}$.

La grève des enseignants a suscité chez nos lecteurs une foule de réactions. Les professeurs ont certes tenu à exprimer leurs points de vue... parfois divergents, d'ailleurs. Mais les parents, et même les jeunes, ont aussi pris la plume. Nous ne pourrons dès lors, à notre grand regret, publier toutes les missives que nous avons reçues. En voilà quelques échantillons. Chacune se fait l'écho, à sa manière, d'autres lettres. Et certaines proposent aussi des pistes à méditer... ${ }^{3}$.

Un deuxième élément expliquant les variations dans le nombre de courriers publiés provient, bien entendu, de la durée des faits. Il est normal que la manifestation des agriculteurs, unique journée de violence à-Bruxelles, fasse couler moins d'encre que la grève des médecins ou celle des enseignants qui ont marqué l'actualité durant plusieurs mois. En outre, la colère des agriculteurs est apaisée dans la semaine par des accords pris au niveau européen. Aussi, la question quitte-t-elle rapidement les feux de l'actualité, rendant les réactions des lecteurs moins pertinentes. Par contre, d'autres événements ne peuvent trouver un règlement rapide, laissant ainsi la question plus longtemps au cœur des débats.

Enfin, certaines grèves affectent le public davantage que d'autres. Que des agriculteurs ou des métallurgistes manifestent peut émouvoir l'opinion, entraîner le saccage de mobilier urbain ou causer des ennuis de circulation. Mais lorsque les gens sont atteints dans leur vie quotidienne, les réactions se font plus vives. Les grèves des services publics, des médecins ou des enseignants seront ainsi souvent mal perçues parce qu'elles toucheront la population dans son quotidien de manière plus sensible que des grèves dans le secteur industriel. L'abondance du courrier s'en ressent inévitablement.

\footnotetext{
1 La Province, le 03/03/82, $\mathrm{n}^{\circ} 51$, p. Mag.2.

2 La Libre Belgique, le 10-11/11/90, $\mathrm{n}^{\circ} 314-315$, p. 2.

3 Le Vif l'Express, le 30/11-06/12/90, n 48, p. 47.
} 


\section{L'identité des lecteurs}

Le "profil" des lecteurs-écrivants est assez difficile à cerner. Jusque dans les années 1990, les informations les concernant restent en effet très parcellaires (1'âge des écrivants n'étant, par exemple, presque jamais mentionné). Elles sont tributaires d'une part, des indications laissées par l'écrivant et, d'autre part, de ce qu'en a gardé la rédaction pour la publication. Certes, tous les journaux exigent un certain nombre d'informations pour prendre en considération les courriers. Aucune lettre anonyme n'est par exemple acceptée. Ainsi, trouvera-t-on régulièrement en tête des rubriques du courrier des lecteurs une note de la rédaction semblable à celle-ci : "Réservée à nos lecteurs, cette rubrique ne publie que des lettres dont l'original porte le nom et l'adresse de l'expéditeur. Toutefois, seules les initiales figurent à la publication. Il n'est tenu aucun compte des lettres anonymes. Rappelons que ce courrier n'engage que nos correspondants occasionnels et que l'insertion d'une lettre ne signifie nullement qu'elle exprime l'opinion du journal"l. Mais si les coordonnées complètes sont exigées par le journal, l'auteur peut cependant demander de ne voir mentionnées dans l'article que ses initiales.

Jusqu'en 1990, les journaux indiquent donc, dans le meilleur des cas, les initiales du lecteur. Les noms sont très rarement mentionnés et encore moins les prénoms. Il est difficile de déterminer si cette situation est le fruit d'une politique déterminée des journaux ou le souhait des écrivants de rester discrets sur leur identité. Quoi qu'il en soit, l'analyse révèle que ce ne sera qu'avec la grève des enseignants que l'on sortira vraiment de l'anonymat. En effet, seuls deux courriers sont alors publiés sans nom. Septante autres présentent l'initiale du prénom suivi du nom du lecteur tandis que près de deux cents affichent nom et prénom. Ajoutons à cela treize groupes d'enseignants et deux de parents dont les noms de chacun ne sont évidemment pas mentionnés mais bien l'établissement ou la localité qu'ils représentent. Bref, il semble désormais que chacun assume pleinement la responsabilité de ses propos ou que la tendance réactionnelle soit à la transparence.

1 Pourquoi Pas?, le 01/04/1971, p. 203. 
Jusqu'en 1971, le lieu d'origine des lecteurs-écrivants n'est pas souvent mentionné1. Mais, à partir de la manifestation des agriculteurs, il est donné dans la majorité des cas ${ }^{2}$. Ainsi, la moitié des interventions qui condamneront la violence de la manifestation émanera de Bruxelles tandis que ceux qui défendent la cause des agriculteurs proviendront essentiellement (mais pas uniquement) de La Province. La même répartition se retrouvera pour les métallurgistes, une de leurs manifestations ayant également dégénéré dans la capitale. Il semble par ailleurs normal que les Bruxellois, marqués en leurs murs et témoins directs des violences, prennent plus volontiers la plume pour réagir à ces événements que ceux qui n'en furent que de "simples téléspectateurs" via la vision du Journal télévisé ou la lecture de leur quotidien. Quant aux grèves des services publics et des enseignants, elles toucheront un large public à travers toute la Wallonie. Les courriers publiés proviendront dès lors de toutes les régions, sans véritable distinction.

Le sexe des lecteurs-écrivants ne peut pas davantage être déterminé. Lors de la grève des enseignants toutefois, le prénom est mentionné beaucoup plus régulièrement et l'on peut ainsi connaître plus facilement le sexe des auteurs. En règle générale, les hommes dominent incontestablement les rubriques du courrier des lecteurs. En ce qui concerne les lettres à propos des manifestations agricoles et métallurgiste, le rapport est particulièrement tranché : une seule femme (sur dix-sept courriers) s'exprime en 1971 et 7 seulement dix ans plus tard pour la grève des métallurgistes. Il est vrai que la teneur des faits concerne un milieu de travail essentiellement dominé par les hommes. On ne peut par ailleurs perdre de vue le fait que, plus généralement, le public d'usagers des journaux quotidiens est majoritairement composé de représentants du sexe masculin. Notons toutefois que les femmes prendront davantage la parole lors des grèves d'enseignants, cette profession étant plus féminisée que le secteur de la sidérurgie. Néanmoins, même lors de cet événement, elles resteront largement minoritaires avec 56 témoignages publiés contre 182 envoyés par des hommes (sans compter le cas des "indéterminés").

\footnotetext{
1 Pour la grève des médecins en 1964 , seuls cinq lieux d'origine sont mentionnés sur 58 courriers.

$292 \%$ des lieux d'origine des lecteurs-écrivants sont mentionnés à partir de 1971.
} 
Reste enfin la profession des lecteurs-écrivants. À nouveau, les conclusions sont peu aisées à tirer car la profession des écrivants n'est pas toujours mentionnée. Elle ne l'est jamais, par exemple, pour les courriers concernant la guerre scolaire ${ }^{1}$. Toutefois, une tendance se dégage : les grévistes et les acteurs directs des mouvements sociaux s'expriment assez peu ${ }^{2}$ alors que ceux qui ont à souffrir de la grève, par contre, prennent souvent la parole. Au risque de caricaturer les situations, on pourrait considérer que les grévistes manifestent leur colère dans la rue tandis que leurs "victimes" occupent les colonnes d'un journal. On peut ainsi considérer que, faute de pouvoir exercer une pression réelle sur une grève, ce mode d'action épistolaire et médiatique constitue un des moyens les plus civils dont dispose la population d'une sociétés industrielle avancée pour exprimer son désarroi, son mécontentement ou son soutien face à un mouvement social. Les citoyens écrivent donc abondamment à leur périodique pour lui faire part de leur expérience personnelle, défendre leurs idées ou exprimer leur rancœur envers les grévistes. Qu'ils soient directement victimes des grèves et des manifestations ou qu'ils n'aient qu'un avis sur la question, beaucoup se sentent concernés par ces mouvements sociaux parce qu'ils les affectent dans leur quotidien. À propos de la grève des médecins, par exemple, les courriers publiés en 1964 seront soit des plaidoyers des médecins contre la Loi Leburton (avec force chiffres pour étayer leur argumentation), soit des témoignages d'experts mutualistes tenant à exposer clairement les implications de la nouvelle loi, soit, et même surtout, des récits de patients prenant parti pour ou contre les grévistes suivant leur expérience heureuse ou malheureuse de la médecine. Ainsi, la grève des médecins mobilisera les lecteurs parce qu'elle les aura atteints dans leur expérience personnelle, au quotidien. Tel praticien défendra son indépendance, tel patient fustigera les spécialistes tandis que tel autre encore soutiendra son médecin traitant.

Par ailleurs, parmi les grévistes, toutes les professions ne réagissent pas de la même façon. Ainsi, sur quinze professions

1 Le manque d'information sur la profession des lecteurs-écrivants crée de temps en temps quelques ambiguittés et pourrait même prêter à confusion. Ainsi, pour la question de l'enseignement, cette lacune ne permet pas toujours de déterminer si le lecteur-écrivant appartient ou non au corps enseignant. Et comme les avis sont partagés au sein même de la communauté éducative, l'analyste est parfois conduit à se demander si l'écrivant est réellement favorable ou opposé à la grève.

2 A l'exception des enseignants, même si $60 \%$ des courriers sur la question proviennent tout de même des parents et personnes étrangères au secteur éducatif. 
mentionnées par les signataires de courriers de lecteurs lors de la grève des médecins, dix exerçaient comme praticiens et justifiaient leur action dans la presse. De même, et encore plus clairement, $40 \%$ des courriers publiés à propos des grèves dans l'enseignement provenaient d'enseignants (grévistes ou non-grévistes) plaidant leur cause et expliquant leurs revendications. Par contre, seuls trois fonctionnaires prirent la parole au sujet des grèves dans le secteur public $^{1}$ et un seul lors du mouvement des métallurgistes. Ce dernier présente d'ailleurs un caractère quelque peu exceptionnel puisqu'il s'agit d'un militant incarcéré suite à la manifestation violente de Bruxelles et qui continue, derrière ses barreaux, à exprimer son opposition au gouvernement ${ }^{2}$. Les professions intellectuelles s'expriment ainsi davantage que les ouvriers ou les agriculteurs, notamment en raison de la teneur de leurs revendications, qui dépasse une simple question pécuniaire pour s'étendre au bien-être des patients et à une meilleure qualité de l'enseignement. Médecins et enseignants tentèrent de rallier leurs patients et les parents à leur cause en démontrant qu'ils agissaient aussi, voire surtout, pour le bien de tous. Motivés par des revendications essentiellement salariales, métallurgistes et fonctionnaires ne peuvent par contre se draper d'arguments aussi généreux et ne prendront dès lors pas autant la parole pour justifier leur action. Mais on ne peut aussi perdre de vue que, bien plus que les agriculteurs ou les métallurgistes, les enseignants sont des gens de lettres. Ce qui peut expliquer l'abondance de leur courrier et son caractère nettement argumentatif.

\section{Les thèmes}

Chaque événement, chaque grève suscite bien sûr des réactions qui lui sont propres ${ }^{3}$. Mais, de 1955 à 1990, réapparaissent régulièrement des thèmes communs aux divers mouvements de grève. Certains propos reviennent inéluctablement comme d'éternels clichés

1 Et parmi ces trois fonctionnaires, celui dont le courrier est publié dans La Dernière Heure témoigne contre ses collèges grévistes et non pour défendre leur cause. (M.C., dans La Dernière Heure, le 03/07/1986, n 184, p. 10).

2 F.R., dans La Libre Belgique, le 20-21/03/1982, nº 78-79, p. 2.

3 L'objet de notre propos n'est évidemment pas d'en dresser ici un inventaire exhaustif. 
traversant la mémoire collective. Cette récurrence dans le contenu des courriers des lecteurs est à ce point frappante qu'ils laissent au chercheur l'impression d'avoir déjà lu ces courriers auparavant et qu'il pourrait dès lors aisément en intervertir les événements. Ainsi, les courriers publiés se divisent-ils clairement en trois catégories inégales : les lettres favorables au mouvement social en cours (39\% des courriers publiés), celles qui y sont opposées (49\%) et celles qui ne prennent pas position à son propos $(12 \%)$.

Contrairement à des événements dramatiques comme l'affaire Dutroux où les lecteurs-écrivants se rangeront globalement derrière un certain consensus, la nature même des mouvements sociaux, qui sépare grévistes et non-grévistes, invite à la polémique et au débat. I1 est dès lors assez naturel que le sujet divise les lecteurs.

\section{Répartition des tendances}

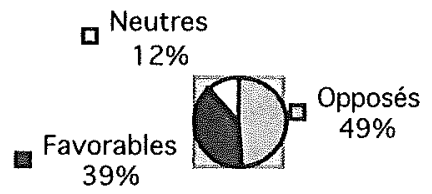

- Opposés 嘈 Favorables aNeutres

\section{Les favorables à la grève}

Nous entendrons par "favorables à la grève" les lecteurs dont les courriers publiés attestent qui adhèrent au mouvement. Qu'ils soient grévistes eux-mêmes ou ne le soient pas, ils prennent fait et cause pour les manifestants et tentent de convaincre autrui du bien fondé de l'action des protestataires. La grande majorité de ces écrivants émane assez naturellement des grévistes eux-mêmes. Médecins désabusés, agriculteurs en colère ou enseignants fatigués, ils prennent la parole pour expliquer leurs revendications et justifier leur action. Toutefois, nous l'avons dit, certains grévistes s'expriment moins que d'autres (essentiellement les cols bleus et les clos blancs). Et, parmi les écrivants grévistes, tous les auteurs de lettres ne se rangent pas du même côté Des voix dissidentes se font en effet entendre au sein même des mouvements. On trouve ainsi, par exemple, bon nombre d'enseignants s'opposant à la grève. A contrario, de simples citoyens, 
que les revendications des grévistes ne concernent pas directement, peuvent prendre la défense des manifestants dans une rubrique du courrier des lecteurs. Globalement toutefois, ces écrivants favorables au mouvement de grève s'avéreront moins nombreux que ceux qui la maudissent, ceux qui ont à pâtir des mouvements sociaux recourant davantage aux supports médiatiques que les grévistes.

\section{Le "triste sort" des grévistes}

Parmi les thèmes récurrents abordés par les lecteurs-écrivants acquis à la cause des grévistes, on relèvera d'abord le chapelet de plaintes énumérées, ainsi que les listes de déclarations compatissantes concernant leur situation professionnelle. Cette argumentation logique peut succinctement se résumer par ces quelques termes: "La condition de cette catégorie professionnelle est tellement lamentable qu'un mouvement de protestations se justifie pleinement. Cela ne peut durer ainsi". Cet exercice littéraire sera particulièrement développé par les enseignants. Au cours du conflit social qui les concerne, il n'est de semaine sans que le courrier de l'un d'eux ne tente de décrire le triste sort d'une profession en mal de valorisation. Les lettres affirmeront que l'on sous-estime leur travail allant bien au-delà des heures prestées en classe et couvrant, par exemple, des prestations aussi diverses que les préparations de cours ou les animations de fancy-fairs ${ }^{1}$. Leurs plaintes revêtent alors des allures de justifications. Elles expliquent que c'est au vu de leur intolérable situation qu'ils ont choisi de faire pression dans la rue lors d'un bras de fer, qui, tout incommodant qu'il soit, n'en demeurait pas moins nécessaire à leurs yeux.

[L'enseignant] est investi de la plus noble tâche (...) et l'institution scolaire ne cesse de l'infantiliser, de le culpabiliser, de faire du maître un exécutant. (...) Infantilisé, l'enseignant doit rendre des comptes à la direction, à l'inspection, aux parents (...). Soumis, il preste des heures supplémentaires gratis pro Deo (...). Exécutant, il applique les directives ministérielles, changeant de méthode, de programme (...).

1 À ce propos, un enseignant parle même, non sans ironie, d'une nouvelle race nobiliaire: "les chevaliers de la fancy-fair". (DE WiTTE-TANCREZ, G., dans Le Soir, le 02/1 1/1990, n² 255, p. 2). 
Enfin, culpabilisé, il le sera parce que l'échec scolaire est "économiquement insupportable" (...) 1 .

La situation vécue sur le terrain par les enseignants, les élèves et les étudiants atteste indubitablement une régression. Disparition du paiement de certaines prestations (conseils de classe, direction de classe...), augmentation des heures prestées, quasi-disparition de certains cours culturels (...), multiplication des participations aux frais scolaires, tombolas, fancy-fairs (...), conditions matérielles de cours à la limite du supportable (sécurité, dégradations...). Si pendant longtemps, les "maîtres" n'ont pas étalé leur misère aux yeux de l'opinion publique, c'est parce qu'ils tenaient à une certaine réserve (...). $C^{\prime}$ est aussi parce que leur capacité de motivation, d'enthousiasme suppléait à bien des manques. Aujourd'hui, bien souvent, le cour n'y est plus, la démotivation s'installe ici ou là, les défections se multiplient, les recrutements se font difficiles ${ }^{2}$.

Dans une moindre mesure, on retrouve le même type d'argumentaire auprès des autres professions en proie à un mouvement social. Ainsi, cette plainte d'un agriculteur : "les paysans sont haïs des habitants des villes qui les prennent pour des grossiers personnages sans culture, trichant avec le beurre et le lait (...)"3. Ou celle de cette fonctionnaire se dit également écœurée par sa situation : "Les agents des services publics dont pas un franc de "maigre" rémunération n'échappe au fisc et qui, de plus, paient depuis plus de 5 ans une lourde cotisation de solidarité, ont, eux, l'impression de... venir en aide au privé. (...) Quand j'entends la plupart des politiciens se gargariser de leurs responsabilités, ça me fait mal aux seins !"4.

Les grévistes ne sont toutefois pas les seuls à prendre la plume pour dénoncer leurs conditions de travail. Dans leurs courriers, il arrive que des "citoyens lambda" prennent également leur défense. Là, un curé expliquera les violences de la manifestation du 23 mars 1971 en écrivant que "la colère du paysan est celle du pauvre homme toujours moqué" ${ }^{2}$. Ici, un patient défendra son médecin traitant, non sans égratigner au passage les politiciens : "On nationalise, on réglemente? Que nos lézards parlementaires travaillent au moins la

1 DueZ-DelPlan R.-M., dans Le Soir, le 31/10/1990, p. 2.

2 EgGermont, M., dans Le Soir, le 28/12/1990, n 302 , p. 2.

3 La Libre Belgique, le 18/04/1971, $\mathrm{n}^{\circ} 103$, p. 2.

${ }_{5}^{4}$ C.L., dans Pourquoi Pas?, le 16/07/1986, n 3529, p. 102.

${ }^{5}$ La Libre Belgique, le 18/04/1971, n 103 , p. 2. 
moitié des heures fournies par le corps médical et nous en reparlerons!"1. Là encore un papa s'opposera même à la reprise des cours et en voudra à l'institutrice de sa fille de ne pas se mobiliser davantage :

C'est avec bien plus que de la réticence que je t'amènerai $\mathrm{A}$... en classe ces lundi et mardi. Tout en reconnaissant la valeur de ton travail ainsi que le «droit au travail» de chacun, je voudrais souligner qu'en tant que père il ne me paraît pas correct de favoriser la tenue des cours, alors que des choses bien plus importantes se jouent pour notre avenir, et pour celui de nos enfants. Face à cela, la perte de quelques journées de cours me semble peser bien peu dans la balance. (...) Ce qui est capital, c'est que l'on nous dit que de plus en plus de parents en ont assez et que l'on fait croire que les perturbations sont devenues intolérables et quotidiennes. Tout cela est archi-faux !?.

\section{Les politiciens (ir)responsables}

De la rédaction de plaintes concernant l'état de leur situation, les grévistes passent volontiers à l'expression de critiques, cherchant ainsi les responsables, désignant les coupables. Leur première cible à ce niveau se situe inévitablement du côté du gouvernement et la classe politique en général. Ceux-ci sont jugés responsables de tous leurs maux et bien souvent taxés d'immobilisme, voire d'incompétence. Le cas de la Loi unique constitue de ceci un exemple éclairant, d'autant plus que les écrivants, non contents de critiquer le gouvernement à propos de cet objet précis, lui reprochent aussi sa mauvaise gestion d'autres dossiers (le Congo et le conflit communautaire). Ainsi, ce commentaire peu amène d'un lecteur-écrivant :

Décidément, «ils» n'en ratent pas une... Après le Congo, la Belgique. Et puis quoi ? (...) Au lieu de s'inspirer de réalités, (...) ils ont, avec un aveuglement administratif, confectionné un cocktail affreux, monstrueux, imbuvable, qu'on veut nous faire absorber de force. Les politiciens ne s'inspirent pas de la réalité et prennent donc des décisions désastreuses. Ce fut le cas pour le Congo. Ca l'est à nouveau pour la loi unique ${ }^{3}$.

1 R.B., dans Pourquoi Pas ?, le 17/04/1964, n 2368, p. 157.

2 BuXANT, G., dans Le Soir, le 24/10/1990, n 248, p. 2.

3 HDVC dans Pourquoi Pas ?, le 13/01/1961, n 2198, p. 111. 
De même, cet autre lecteur n'épargne pas non plus le gouvernement :

Enfin il n'y a pas que la loi unique. Et la bienveillance envers les inciviques? Et la chinoiserie linguistique? Et le désastre congolais ? (...) La grève est légitime. C'est en effet toujours sur la classe ouvrière que retombent les conséquences de la politique imbécile d'un gouvernement qui s'est déjà fourvoyé pour le Congo et le conflit communautaire'

Et un autre lecteur de conclure :

Les grèves ont certes leurs excès mais elles expriment la révolte d'une frange importante de la population (les travailleurs) que nos gouvernants feraient bien de prendre en compte $^{2}$.

Les grévistes égratignent sans relâche une classe politique qualifiée de "bons à rien", qui se moque d'eux, se réfugie derrière de fausses excuses et n'assume pas ses responsabilités. À ce sujet, les enseignants font preuve d'une imagination débordante pour pourfendre leurs ministres et s'en moquer. Ici, c'est une lettre qui décline sur tous les tons les revendications des enseignants, selon la célèbre tirade du "nez" de Rostand". Là, c'est une chanson d'un professeur sur l'air connu "Bonbons, caramels, esquimaux, chocolats"4. Plus tard, La Dernière Heure publiera encore ce courrier envoyé par cinq enseignants, parodie du "Notre Père" critiquant le ministre Yvan Ylieff :

Père Ylieff, qui es au ministère, que ta paie soit augmentée, que ta volonté soit faite dans le libre comme dans l'officiel, que ton règne s'étende aux profs comme aux élèves. Donnenous aujourd'hui notre grève quotidienne. Pardonne-nous nos revendications comme nous te pardonnons aussi pour nos locaux délabrés. Ne nous soumets pas aux horaires perturbés et délivre-nous des restrictions budgétaires. Amen"5.

\footnotetext{
1 S.H., dans Pourquoi Pas?, le 13/0//1961, n 2198, pp. 111-112.

2 E. D., dans Pourquoi Pas ?, le 27/01/1961, n ${ }^{\circ} 2200$, p. 103.

3 Claude, C., dans Le Vif l' Express, le 23-29/11/1990, n 47, p. 225.

4 BarbiauX, G., dans Le Soir, le 28/12/1990, n 302, p. 2.

5 La Dernière Heure, le 28/1 1/1990, $\mathrm{n}^{\circ}$ 332, p. 16.
} 
Mais si certains lecteurs-écrivants se déchaînent volontiers contre les politiciens, les points de vue peuvent changer radicalement selon la couleur du gouvernement en place au moment des événements, la catégorie professionnelle concernée et la tendance des journaux qui publient ces courriers. En effet, si certains journaux proposent sans préférence des avis divergents, d'autres favorisent leur point de vue en publiant des courriers qui vont, pour la plupart, dans le sens de leur ligne idéologique et rédactionnelle!. Ainsi, des courriers virulents contre le gouvernement seront-ils davantage l'apanage de la presse de gauche quand il s'agit de conflits ouvriers, comme le montre cet extrait publié dans Le Peuple:

Ces propositions intéressantes qui permettraient de sauver la sidérurgie par «l'aval» seront-elles, comme les autres, mises au tiroir par les ministres et les pouvoirs «compétents», dévorés par leurs jalousies respectives, paralysés par le mépris flamand et stoppés par l'administration des incompétences et des forces corruptrices ??

Par contre, les courriers publiés dans la presse socialiste soutiendront le gouvernement en 1964, car la loi Leburton qui provoque l'ire des médecins émane d'un ministre socialiste. Le vrai sera évidemment tout aussi vrai... Lors de la grève des services publics par exemple, les lecteurs-écrivants de la presse de droite attribueront la mauvaise situation du secteur à la gestion dispendieuse des socialistes depuis les années 1960.

Depuis plus de 40 ans, les socialistes et syndicalistes, incapables de gouverner lorsqu'ils sont au pouvoir, grévistes destructeurs dans l'opposition, prodigues de l'argent des contribuables, n'ont cessé de conduire le pays à la ruine ${ }^{3}$.

Cette critique des socialistes constitue un refrain régulier, exploité surtout dans La Dernière Heure, comme l'illustrent ces deux lecteurs: "MM. Mathot, Spitaels et Debunne, n'ayant pas réussi à avoir la majorité aux dernières élections, ont décidé de descendre dans

I Soit qu'ils effectuent un tri dans ce sens, soit que leur lectorat, majoritairement acquis à la ligne du journal, lui envoie davantage de courriers de ce type. Cela reste impossible à cerner...

2 A. Yerna, dans Le Peuple, le 01/03/1982, nº 63, p. 5.

3 R. P., dans La Dernière Heure, le 28/07/1986, n 209, p. 8. 
la rue, comme lors de la loi unique. Cette fois, nous assistons aux mêmes tripotages"'.

Le PS et la FGTB, exhibant sans pudeur leur complicité imbécile et criminelle, poussent leurs troupes d'irresponsables à multiplier les grèves suicidaires qui risquent de rendre inopérants les sacrifices déjà consentis par la population pour tenter d'améliorer le triste bilan du passé. (...) Nous refusons obstinément de constater que le tandem PS-FGTB complote chaque jour pour assassiner gaiement la liberté!?2.

Notons toutefois que le poids des idéologies sera davantage prégnant dans les années 1950 et 1960 que dans les années 1990. Il s'estompera progressivement avec l'effritement de la pilarisation. Ainsi, les deux conflits ayant surgi autour de questions liées à l'enseignement présentent-ils un profil foncièrement différent. Lors de la guerre scolaire, chacun se sent concerné dès lors qu'il défend certaines convictions politiques. Il n'est pas nécessaire pour cela d'avoir son enfant à l'école. Le débat se situe clairement sur le plan des opinions politiques entre catholiques et anticléricaux : on défend une idéologie et un parti derrière un type d'école. Les uns prennent la défense des catholiques contre la loi Collard et les socialistes en général : “

Un ami, lui aussi socialiste acharné, a combattu l'école catholique jusqu'au jour où sa jeune fille de vingt ans, devenue folle, il a cherché en vain un institut officiel pour placer son enfant : il n'y avait que des calotins imbéciles et profiteurs pour s'en occuper"3.

Les autres, par contre, pourfendent les catholiques derrière le réseau libre :

Libéral de veille souche, mon premier et mon plus grand principe est la liberté d'opinion. (...) Et voilà la raison pour laquelle je m'insurge avec véhémence contre l'esprit partisan et sectaire du PSC en général et de l'épiscopat en particulier. Une pareille mauvaise foi vous fait bondir et l'on comprend, vis-à-vis d'un tel comportement à notre époque de liberté, ce qui a pu se passer en Espagne durant l'Inquisition quand

\footnotetext{
1 M. W., dans La Dernière Heure, le 17/03/01982, n 76, p. 7.

2 W. C., dans La Dernière Heure, le 19/06/1986, n 170, p. 10.

3 M. C., dans Pourquoi Pas?, le 24/12/1954, n 1882, p. 94.
} 
moines et religieux régnaient en souverains maîtres ! Et puisque, pour ces fanatiques, l'école officielle est le repaire de Satan, (...) attendons-nous, si on les suivait sur ce chemin, à ce que, si un jour ils obtenaient une majorité suffisante, ils ne décrètent qu'il doit y avoir une armée neutre et une armée libre (entendez catholique) ${ }^{\mathrm{l}}$.

Lors de la grève des enseignants en 1990, le débat est tout autre. Il s'articule autour d'enjeux éducatifs et mobilise avant tout des parents inquiets pour l'avenir de leurs enfants. Le temps a relégué au second plan les combats idéologiques pour faire place à des points de vue plus personnels, détachés des considérations d'ordre politique. La véritable question s'avère désormais relever du débat de société, et du lien entre la qualité de l'avenir promis aux enfants et celle de l'enseignement qui leur est prodigué.

Parents, combien de temps encore laisserons-nous nos enseignants défendre SEULS nos enfants ? (...) Quand allonsnous comprendre que ce sont ces citoyens-là qui communiquent le SAVOIR aux adultes du XXIe siècle ? ${ }^{2}$

C'est pour sauver l'école et la qualité de l'enseignement pour tous que les professeurs se battent. Il y va de l'avenir de vos enfants et des générations futures. Il s'agit d'un problème de fond bien plus vaste que de simples revendications salariales"3.

Les ministres se rendent-ils compte que, depuis le mois de mai, nos enfants ne vont plus régulièrement à l'école et qu'ils n'y vont même plus du tout depuis le mois d'octobre ? (...) Savent-ils que, depuis lors, nos enfants, appelés pourtant à former l'avenir de notre société, sont livrés à eux-mêmes et errent dans les rues en ne sachant que faire ?4

Si, au départ, j'ai suivi avec beaucoup de sympathie le mouvements des professeurs, il n'en est plus de même actuellement et je me permets de protester quant à la manière désinvolte dont les professeurs se préoccupent dans les faits de l'avenir de nos enfants. Ceux-ci sont en train d'accumuler un

1 E. B., dans Pourquoi Pas?, le 04/03/1955, $\mathrm{n}^{\circ} 1892$, p. 67.

2 Safarian, J., dans Le Vif l'Express, le 16-22/11/1990, $n^{\circ} 46$, p. 237.

3 Un groupe de professeurs de l'Athénée d'Uccle 2, dans Le Soir, le 31/10/1990, $\mathrm{n}^{\circ} 254$, p. 2.

4 TRIL, dans La Dernière Heure, le 28/11/1990, nº 332, p. 16. 
retard considérable, et cela me préoccupe particulièrement pour les élèves en classes terminales ${ }^{1}$.

\section{La justification des dérapages}

Enfin, après les plaintes et les critiques contre le monde politique, on relève -en moindre nombre- dans les thématiques du courrier des lecteurs divers types des justifications des dérapages survenus lors de certaines manifestations. À ce titre, trois cas présentent des similitudes frappantes: les manifestations des agriculteurs le 23 mars 1971, celle des métallurgistes le 16 mars 1982 et celle des enseignants le 27 septembre 1990, les deux premières ayant fait couler beaucoup d'encre. À près de dix ans d'intervalle, on relèvera dans les lettres publiées des arguments presque identiques, tenant en peu de mots : les grévistes en colère ne sont pas les vrais fautifs des troubles mais bien les forces de l'ordre ou les "spectateurs" qui ont scandaleusement provoqué les manifestants.

En 1971, une lectrice de La Libre Belgique, propriétaire terrienne, parle ainsi de la "provocation de certains Bruxellois qui ont lancé des tomates pourries sur les manifestants et les ont traités de paysans"2. Le même prétexte sera avancé par les métallurgistes :

Vous, premier gardien de la Justice, avez-vous fait ouvrir une enquête sur l'agression commise par vos sbires, le jour de la manifestation sur un pauvre type couché par terre et tabassé, par 5 ou 6 pandores armés de matraques encore insuffisantes car les coups de pieds suivaient (...). Ils ne sont pas seulement passibles d'agression mais aussi de refus d'aide à personne en danger ${ }^{3}$.

En 1990, le renversement entre "agresseurs" et "agressés" sera une nouvelle fois exposé par un enseignant :

Valmy Féaux est content (...). Jamais fête ne fut plus réussie : les invités étaient nombreux et furent reçus en grande pompe. (...) C'est alors qu'on vit surgir d'un peu partout des autopompes affolées, des régiments décidés, un hélicoptère inquiet qui cherchait désespérément le sens de la marche : la

1 VerSCOORE, L., dans Le Soir, le 08/11/1990, n² 260, p. 2.

2 La Libre Belgique, le 18/04/1971, n 103, p. 2.

3 MASSON, L., dans Le Peuple, le 09/04/1982, n 99, p. 5. 
provocation mise en scène. Ce n'était donc plus la fête ? Évidemment (...) rien n'est plus dangereux que l'intelligence dans la rue. C'est qu'elle fait peur : l'état-major perd le nord et recule $(\ldots)^{1}$.

Certains grévistes se retranchent donc derrière la provocation pour justifier les violences. Mais cet argument est rapidement contesté par les non-grévistes. Car, en effet, les défenseurs des grèves comptent évidemment leurs détracteurs. Plus nombreux, ceux-ci recourent fréquemment à la rubrique du courrier des lecteurs pour exposer leur point de vue. Et bien des arguments évoqués par les grévistes trouvent chez eux une réponse. Les uns se plaindront de leurs mauvaises conditions de travail ; les autres, tout en étant d'accord avec leurs revendications, s'indigneront de leurs méthodes. Les uns critiqueront les politiciens; les autres pourfendront les syndicalistes. Les uns reprocheront aux gendarmes leur comportement provocateur ; les autres condamneront les excès et les violences des manifestations. Double facette d'un même enjeu, double regard sur une même situation.

\section{Les opposés à la grève}

\section{La critique des méthodes}

Si une partie de ceux qui s'expriment dans le courrier des lecteurs s'opposent à la grève, c'est avant tout parce qu'ils n'en acceptent pas les méthodes. En effet, beaucoup de victimes des grèves reconnaissent que les revendications des grévistes sont justifiées et méritent attention. Mais ils ne peuvent toutefois se solidariser à un mouvement dont ils condamnent les moyens d'action. Ainsi, les critiques adressées aux médecins concerneront surtout leur abandon des patients. D'aucuns ne supporteront pas l'idée d'un corps médical en grève, estimant qu'il est de son devoir de veiller sur la santé de ses concitoyens. Ils considéreront les revendications des médecins peutêtre justes mais leur méthode "immorale".

Dix mille hommes en colère ? Peut-être. En tout cas, 10.000 hommes qui se sont déconsidérés. Dix mille hommes qui ont

1 Delgleize, P., dans Le Soir, le 04/10/1990, n 231, p. 2. 
failli à leur serment, failli à leur devoir. Un médecin a tous les droits, hormis celui d'abandonner ses malades. (...) Si les revendications sont légitimes, le moyen utilisé pour les imposer est détestable. Il est incroyable que le gouvernement n'ait pas immédiatement répliqué à la grève par la réquisition et Le Rappel militaire'.

Je ne veux pas prendre position quant au bien-fondé éventuel du refus, par les médecins, des obligations que la loi contestée leur imposait. Ce que je dénonce, c'est le principe même d'une grève de gens, qui (ayant librement choisi une profession) ABANDONNENT leurs malades ${ }^{2}$

L'illégitimité des méthodes se retrouve évoquée dans le cas de quasiment tous les conflits analysés lors de cette étude. En effet, elle est aussi contestée pour les agriculteurs, les métallurgistes, les fonctionnaires et les enseignants, le même discours revenant inlassablement. Tout comme l'abandon du patient, la "prise d'otage" des enfants livrés à eux-mêmes ou les violences lors des manifestations constituent autant de méthodes jugées "intolérables" finissant par désolidariser la population de la cause des grévistes.

En 1971, un lecteur-écrivant de Bruxelles, se montre ainsi particulièrement dur à l'égard des agriculteurs.

Permettez à un travailleur manuel, à un ouvrier de la capitale, de vous exprimer son écœurement et son profond dégoût de méthodes que nous, au plus fort de l'agitation sociale en 1960 , avons répugné à utiliser. Sachez que tout le monde vous juge sévèrement (...). S'il est de votre droit le plus strict de proclamer ouvertement votre mécontentement, il vous est par contre dénié de vous livrer à des actes que certains documents, filmés en 40-45, montraient comme étant l'apanage de la soldatesque nazie en pays conquis ${ }^{3}$.

La même idée est reprise contre les méthodes des métallurgistes en 1982 :

Je ne conteste en aucune manière le droit de manifester ses opinions (...). Il n'empêche qu'il est inadmissible que des voyous et des casseurs profitent de l'impunité qui leur est garantie pour se permettre de saccager tout un quartier (...) Je

1 P.S., dans Pourquoi Pas ?, le 17/04/1964, n² 2363, p. 151.

2 R.C., dans Pourquoi Pas? , le 17/04/1964, n² 2363, p. 152.

3 R.B., dans Pourquoi Pas?, le 01/04/1971, n 2731, p. 302. 
crois qu'il est temps que se forme une réaction contre ces violations répétées de nos droits de citoyen (piquets de grève...) $)^{\mathrm{l}}$.

La violence ne requiert que peu de génie et n'est que trop l'acte insensé d'un lâche se couvrant de l'anonymat des masses ouvrières en lutte pour leur juste cause sociale. (...) Il y a lieu d'appliquer la théorie d'Hippocrate "d'abord ne pas nuire" au corps social que l'on veut défendre, nous aurons ainsi des grèves "sympa" qui n'atteindront plus les innocents, qui assureront et la liberté individuelle de travail et l'appui inconditionnel de la population. ${ }^{2}$.

Principal grief contre les grévistes du secteur public (et quasi unique objet des courriers publiés sur la question), les méthodes des protestataires sont considérées comme un non-sens, une "erreur monumentale" car, inadaptées, elles touchent la mauvaise cible : le public. Un lecteur, par exemple, explique aux grévistes :

Une grève, et surtout dans ce cas précis, n'est plus un moyen de pression mais tout au plus une recherche de publicité aux frais uniquement de la population et même à vos propres frais, mesdames et messieurs les grévistes. En fait de publicité, là, vous avez atteint votre but, mais, croyez-le bien, pas en votre faveur ${ }^{3}$.

De inême, un autre déclare :

Je m'associe pleinement (..) au mécontentement des fonctionnaires à l'encontre de nos dirigeants. Mais les grèves se trompent de cible. C'est le citoyen qu'elles ennuient et non pas ceux qui sont à l'origine des mouvements d'humeur des grévistes ${ }^{4}$.

1 Delacroix, Y., dans La Libre Belgique, le 24/03/1982, nº 83, p. 2.

2 A.V.D., dans $L a$ Cité, le 29/03/1982, n 74, p. 2.

3 VANDENBosCh, B., dans Le Soir, le 02/06/1986, $\mathrm{n}^{\circ} 126$, p. 22 . Ce courrier est publié dans trois périodiques, le texte présentant quelques légères différences d'une version à l'autre. (Aussi dans Le Vif l'Express, le 23-29/05/1986, n 170, p. 6. et dans La Dernière Heure, le 11/06/1986, $\mathrm{n}^{\circ} 162$, p. 10.) Notons à ce propos que, lorsque des journaux différents publient des courriers communs, ils effectuent chacun des coupes qui leur sont propres et publient parfois des extraits différents d'un même courrier. Ainsi peut-on aussi constater que certains journaux apportent de très légères modifications aux textes des lecteurs.

4 Brumagne, A., dans Le Soir, le 02/06/1986, n 126, p. 22. 
Enfin, les enseignants, jugés inciviques et irresponsables, sont eux aussi vivement critiqués pour leur "prise d'otage" des élèves, désormais livrés à eux-mêmes. Les parents surtout, mais aussi certains enseignants dissidents, prennent la plume pour dénoncer leur procédé inefficace, inadapté et indigne. "Impardonnable, écœurant, inique, odieux..." sont des mots utilisés pour définir une grève qualifiée même "d'assassinat". Et, le mouvement est d'autant plus réprouvé que les enseignants continuent d'être rémunérés durant ces grèves. Ainsi, la méthode est non seulement perçue comme irresponsable, mais en plus les grévistes sont estimés ne pas en assumer les conséquences salariales. Au fil des courriers, on trouvera même de la sorte des parents portant symboliquement plainte, via le journal, contre les enseignants qui n'accomplissent pas leur devoir. Même si les revendications sont estimées, justes mais le recours à la grève est, lui, jugé inadmissible par beaucoup.

J'ai toujours eu le plus grand respect pour le corps enseignant (...). Même si l'on admet certaines de leurs revendications, on ne peut pas leur emboîter le pas dans la méthode qu'ils emploient pour y arriver. (...) Si les enseignants veulent voir aboutir leurs revendications qu'ils s'en prennent directement à ceux qu'ils croient être la cause de tous leurs maux (...) mais qu'ils gardent leur rôle d'éducateurs et de formateurs et non de révolutionnaires comme certains piquets de grève pour qui le mot démocratie n'a pas de sens ${ }^{2}$.

Mesdames et messieurs les enseignants, peut-être vos revendications sont-elles fondées et votre "combat" légitime à vos yeux. Ce qui l'est moins, c'est votre démission de la tâche sacrée qui vous incombe : veiller à l'éducation de cette fragile catégorie d'être humains en pleine formation $(. . .)^{3}$.

Qu'il soit permis d'exprimer et de justifier ici un point de vue qui est opposé à la poursuite d'une grève dont les enfants sont les victimes. Trois choses heurtent le bon sens et l'équité : le fait que les jours de grève soient payés aux enseignants grévistes est en contradiction avec la plus élémentaire équité. En saine démocratie les grévistes y vont de leur poche. Le fait que ce soient les enfants qui soient pris en otage (méthodes dictatoriales). Le préalable des enseignants qui s'opposent à

1 Dumont, D., dans Le Soir, le 24-25/11/1990, n² 274, p. 2.

2 DanoIs, G., dans La Libre Belgique, le 29/11/1990, $\mathrm{n}^{\circ} 333$, p. 11.

3 HoYoIs, E., dans Le Soir, le 24/10/1990, n² 248, p. 2. 
une restructuration souhaitée par chacun mais qui pourrait toucher à la sécurité de l'emploi. À trop vouloir, on perd tout crédit $^{1}$.

Certains enseignants eux-mêmes se mobilisent contre leurs collègues grévistes :

Nous n'approuvons pas les déclarations maladroites du ministre à notre égard pas plus que les mesures financières proposées, mais nous sommes opposés à ces grèves malsaines, archaïques et aberrantes qui lèsent l'enfant et l'étudiant et exacerbent à juste titre les critiques contre notre profession ${ }^{2}$.

Un deuxième grief, lié au premier, revient invariablement dans les critiques adressées aux grévistes. Selon les auteurs des lettres publiées, ils ne se contenteraient pas de ne pas atteindre la bonne cible. En plus, ils remettraient en cause la liberté au travail de leurs collègues non-grévistes, les privant ainsi d'un droit imprescriptible. Certaines lettres en viennent même dès lors à mettre en doute le réel qu'aurait réellement recueilli le mouvement s'il n'avait pas revêtu un aspect forcé : qui prouve que la grève aurait connu une telle ampleur si certains employés n'avaient été "invités" par leurs collègues à rester chez eux ? Alors que cet argument s'avère inexistant pour les grèves contre la loi unique, pour le mouvement des médecins en 1964 ou pour la manifestation des agriculteurs, la polémique fait par contre rage lors des grèves des métallurgistes et surtout des enseignants. À tel point que, outre les parents révoltés, certains professeurs nongrévistes dénoncent également les pressions que d'autres collègues exercent sur eux. Beaucoup de lecteurs-écrivants s'attachent dès lors à démontrer le caractère illégal des piquets de grève, symbole de la dictature des syndicats.

Le slogan "droit de grève mais liberté de travail" commence un peu tard à être repris clairement et avec fermeté par l'opinion publique, la presse, les médias. Or, la liberté du travail et la liberté tout court ne pourront être acquises que quand les piquets de grève seront mis hors-la-loi et condamnés. (...) Je crois également qu'il serait grand temps que la "Ligue des droits de l'homme", qui est si prompte à s'occuper des droits bafoués dans les pays lointains, se penche

1 Dupret, E., dans La Libre Belgique, le 29/11/1990, n³33, p. 11.

2 Fourneau, A. M., dans La Libre Belgique, le 10-11/11/1990, n³14-315, p. 2. 
sur nos propres problèmes et dénonce les façons scandaleuses d'agir des syndicalistes ${ }^{1}$.

Quand la FGTB installe des piquets de grève, elle abuse de la démocratie en déniant à ceux qui souhaitent continuer à travailler la possibilité constitutionnelle d'exercer leur droit au travail (...). Il serait souhaitable (...) que les autorités usent des pouvoirs qui leur ont été donnés pour décréter enfin que les piquets de grève sont illégaux (...). Alors, mais alors seulement, nous aurions le droit de parler sans rigoler de démocratie, de liberté et d'égalité de tous devant la loi $!^{2}$.

Mais si la loi permet la grève, elle protège aussi le droit au travail. Alors, pourquoi ces piquets de grève ? (...) Le droit au travail est donc refusé à ceux qui souhaitent travailler. C'est donc un exemple d'incivisme et de non-respect des lois qui est donné aux enfants ${ }^{3}$.

Ces trois extraits, publiés à quatre ans d'intervalle et pour des événements différents (grèves des métallurgistes, des fonctionnaires et des enseignants), présentent de réelles similitudes tant sur le fond (mêmes arguments : les piquets de grève qui portent atteinte à la liberté au travail devraient être mis hors-la-loi) que sur la forme (même vocabulaire utilisé : droit au travail, piquets de grèves illégaux...).

Par ailleurs, la critique des méthodes employées peut prendre des formes très différentes selon le point de vue politique de celui qui l'énonce. Ainsi, pour la grève des métallurgistes, les rubriques du Peuple et de L'Écho de la Bourse présentent-elles des courriers totalement opposés mais critiquant tous deux les méthodes des grévistes. On trouvera dans le journal socialiste la lettre d'un lecteur soutenant la grève mais lui reprochant ses trop douces méthodes. Dans un langage assez vulgaire, l'auteur de la lettre invitera même ses compagnons protestataires à passer à l'action et annoncera ainsi les violences du 16 mars. "Allez-vous attendre la venue d'un nouveau Degrelle et de ses chiens galeux, en les laissant profiter d'un nouveau merdier ?" Et, comparant la grève de 1982 avec l'échec de celle de 1960 : "Aujourd'hui on remet ça au coup par coup avec les mêmes formes, les mêmes pèlerinages inutiles, alors... NON... cent fois...

1 C.B., dans La Libre Belgique, le 20/04/1982, $n^{\circ} 110$, p. 2.

2 W.C., dans La Dernière Heure, le 19/06/1986, n 170 , p. 10.

3 La Nouvelle Gazette, le 27/11/1990, n²76, p. 4. 
NON. Le temps des sommations est passé. Seul l'action en force peut encore être payante" '. Ton et idées très différents, d'autre part, mais point de vue (davantage attendu), également critique du principe de la grève dans l'Écho ou dans La Libre Belgique qui rendent ainsi compte du point de vue patronal. Dans ces cas, les courriers publiés dénonceront vivement la grève des métallurgiques parce qu'elle sape l'économie belge, repousse les investisseurs étrangers et fait échouer la récente dévaluation du franc belge.

Indifférents à la santé économique et financière du pays, peu soucieux du sort de notre franc, qui après trois semaines de dévaluation bat déjà de l'aile par leur seule et unique faute, ils ne comprennent rien à rien² .

Ainsi l'image de notre pays à l'étranger sera celle des grèves et des manifestations tournant à l'émeute. Cela ne va pas convaincre l'investisseur étranger de risquer ses capitaux dans nos contrées ${ }^{3}$.

\section{La "dictature" des syndicats}

Les vifs reproches que l'on trouve exprimés par le courrier des lecteurs contre les méthodes des grévistes s'expriment avec virulence à propos les instigateurs des grèves, à savoir les responsables syndicaux. Ceux-ci font en effet l'objet de critiques pour leur comportement considéré comme "dictatorial". Dans La Libre Belgique, un lecteur affirme "qu' on n'a pas fini de se plaindre de l'influence des syndicats et de leurs méthodes de chantage et d'intimidation"4. En 1990, une enseignante, tient des propos désabusés allant dans le même sens :

Une refonte de tout le système scolaire s'impose pour affronter l'avenir. Etait-il, pour autant, nécessaire que les syndicats utilisent des méthodes d'intimidation proches du totalitarisme (...) pour écraser une forte minorité d'enseignants défavorables au mouvement ? (...) La fin justifie les moyens. Je pensais que

1 VAN Dorn, R., dans Le Peuple, le 13-14/03/1982, nº 72-73, p. 6.

2 G. P., dans L'Echo de la Bourse, le 19-21/03/1982, n 55, p. 15.

3 H.P.O., dans La Libre Belgique, le 15/04/1982, n ${ }^{\circ} 105$, p. 2.

4 H.B., dans La Libre Belgique, le 13-14/03/1982, n 72-73, p. 2. 
la devise de Machiavel avait vécu sous nos latitudes démocratiques. Actuellement, le doute s'est emparé de moi ${ }^{1}$..

Et un autre lecteur-écrivant d'ajouter :

Le syndicat est une machine administrative vieillie, vivant aux crochets des ouvriers et dont les dirigeants exploitent la naïveté de certains, exigent la participation d'autres (...) En plus de 30 ans de métier, je n'ai jamais vu d'intervention des syndicats que pour la protection de paresseux, de carottiers, de saboteurs et même de voleurs².

Pour certains lecteurs-écrivants, les syndicats imposent les piquets de grèves. Ils incitent leurs troupes à la violence. Et surtout, ils n'assument pas les responsabilités des débordements de leurs affiliés. Lors de la manifestation des métallurgistes en particulier, ils sont pointés du doigt comme les agitateurs, les fauteurs de troubles qui ont fui lâchement la scène des affrontements lorsque les événements ont dégénéré. De nombreux auteurs demandent donc que l'on mette fin à l'irresponsabilité légale des syndicats en leur donnant une personnalité juridique. Cela permettrait de les poursuivre pénalement pour les violences commises par leurs troupes.

Quand ce ne sont pas les agriculteurs ce sont les métallos qui éprouvent le besoin sordide non seulement de manifester à Bruxelles en y perturbant toute l'activité commerciale, mais bien souvent de tout casser sur leur passage. (...) Ces organisateurs de manifestations (qui s'éclipsent dès que cela tourne mal) ne pourrait-on pas les rendre responsables des dégâts qu'ils provoquent?

Véritables coupables aux yeux de la presse conservatrice, ils sont durement jugés par ses lecteurs-écrivants. Selon eux, les syndicats, soutenus plus ou moins dans l'ombre par la "clique socialiste", ne sont que des meneurs irresponsables qui conduisent le pays à la ruine. "J'accuse la FGTB et son complice de toujours, le PS, de manque de civisme, d'irresponsabilité caractérisée, de complot organisé contre le devenir d'un pays que leur soif de pouvoir pousse délibérément vers la faillite"3. Et un dernier auteur de conclure avec emportement :

\footnotetext{
MinET, A., dans La Dernière Heure, le 22/11/1990, n 326, p. 11.

BoulLLET, J., dans La Province, le 03/03/1982, n 51, p. Mag. 2.

3 W.C., dans La Dernière Heure, le 19/06/1986, nº 170, p. 10.
} 
Comment ne pas être scandalisé par ce qui se passe depuis début mai principalement en Wallonie ! (...) Personnellement, j'appelle cela du TERRORISME et ces messieurs déstabilisateurs ne valent guère mieux que les CCC. (...) Quand j'entends des dirigeants syndicaux déclarer à la RTBF : «Nous sommes très satisfaits. La Wallonie est paralysée», j'ai envie de leur crier : ASSASSINS ! ${ }^{1}$.

\section{La partialité de la RTBF}

Enfin, après la critique des "méthodes" et de l'irresponsabilité des dirigeants syndicaux, un autre thème se présente de manière récurrente dans les courriers publiés par certains organes de presse : celui de la partialité de la RTBF. Dans les lettres de lecteurs, en effet, la mise en cause exprimée à l'égard des médias ne se manifeste pas fréquemment à l'égard de la presse écrite, mais presque exclusivement contre l'entreprise audiovisuelle publique. On aurait pu attendre de la rubrique du courrier des lecteurs qu'elle accueille les feedbacks des lecteurs sur leur journal mais tel ne semble pas être le rôle que lui assignent les écrivants. La rubrique du courrier des lecteurs n'est donc pas précisément un lieu de médiation ni de rétroaction. Si des rectifications sont publiées, elles se font davantage réponses aux arguments avancés par un autre lecteur que par le journal lui-même. Notons toutefois que La Libre Belgique publie une lettre de protestation contre ses prises de positions. Cette lettre, reproduite en dehors de la rubrique classique du courrier des lecteurs, est de la main de l'administrateur général de la RTBF, Robert Wangermée. Celui-ci s'insurge contre les propos tendancieux de $L a$ Libre Belgique à l'égard de la chaîne publique.

Même si c'est avec un certain retard, je voudrais revenir sur une mise en cause de la RTBF que vous aviez publiée dans votre édition du 18 mars concernant la manifestation organisée à Bruxelles par les sidérurgistes. Vous avez, en effet, accusé le journal télévisé d'avoir «tronqué les faits» en diffusant une scène où l'on voyait des gendarmes frappant un manifestant à terre. (...) La gravité des accusations que vous avez formulées contre la RTBF m'incite, malgré le retard, à vous demander de donner connaissance à vos lecteurs, de cette mise au point ${ }^{2}$.

1 M.K., dans Pourquoi Pas?, le 09/07/1986, n 3528, p. 101.

2 WANGERMÉE, dans La Libre Belgique, le 07/04/1982, n 97, p. 2. 
Dénoncée par la presse non socialiste, le parti pris de la RTBF en faveur des grévistes et des manifestants exaspère bien des lecteurs écrivant dans des journaux conservateurs. L'Écho de la Bourse publie ainsi quelques courriers contre la chaîne publique.

La gangrène socialiste, dictatoriale en Wallonie, continue d'envahir la RTBF, qu'un Wallon sur deux paie pour entendre les âneries des Spitaels, Coëme et autres «profiteurs du peuple». (...) On a parfaitement raison de vouloir mettre fin aux excès bureaucratiques du million de fonctionnaires «publics» qui desservent (au lieu de servir) la masse silencieuse qui en a par-dessus la tête! Alerte !!

La Dernière Heure, toutefois, sera l'organe de presse dans lequel ce thème sera particulièrement exploité2 " "Ce nouveau tour de passepasse confirme une fois de plus que notre RTBF est depuis bien longtemps, non seulement devenue la RTBFGTB, mais aussi le fief de la gauche. (...) Quand, les francophones qui n'appartiennent pas à la Wallonie ou qui ne sont pas de la gauche auront-ils leur TV ?"3. Et un lecteur de déclarer, péremptoire : "Il faut mettre hors d'état de nuire ces obsédés de la RTBF payés pour détruire notre jeunesse et soutenir les syndicats"4.

Ces mots montrent combien les positions peuvent être tranchées et le débat passionné au sein d'une presse qui, pendant une part importante de la période considérée, était encore vecteur d'opinions et d'engagements politiques affirmés, souvent confortés par la tonalité des lettres reproduites dans la rubrique de courrier des lecteurs.

Cependant, il faut ajouter que cette rubrique peut aussi se faire l'écho de points de vue moins engagés.

1 A.I., dans L'Echo de la Bourse, le 23-25/05/1985, n 98, p. 14.

2 La première fois que La Dernière Heure publiera, dans ce qu'elle affirme être son entièreté, le courrier d'un de ses lecteurs, ce sera pour dénoncer la partialité de la RTBF dans son traitement de l'information sur la violente manifestation des métallurgistes. La rédaction introduira d'ailleurs la lettre en ces termes : "Nous recevons de M. R.D. une longue lettre dans laquelle sont relevés et analysés les dernières «impartialités» de la RTBF. Cette lettre ne peut être tronquée. Nous la publions donc dans son texte intégral". (R.D, dans La Dernière Heure, le 0304/04/1982, no $93-94$, p. 7.$)$

3 E.V., dans La Dernière Heure, le 29/03/1982, $n^{\circ} 88$, p. 8.

4 A.H., dans La Dernière Heure, le 05/04/1982, n 95 , p. 7. 


\section{Les "neutres"}

Parmi la multitude de lecteurs-écrivants, il reste une catégorie minoritaire mais non négligeable pour autant : celle les "neutres", qui ne prennent aucun parti, mais tentent de dépassionner le débat. En retrait des polémiques, ces "sages" cherchent à comprendre l'origine du malaise (sans condamner l'un ou l'autre camp) et à y apporter de justes solutions. Leur propos, plus détaché mais sérieux, consiste donc à suggérer des pistes de réflexions pour sortir pacifiquement de l'impasse et comprend autant des recommandations pour améliorer les conditions de travail des grévistes que des conseils pour que les revendications exprimées atteignent la bonne cible. De la guerre scolaire en 1955 à la grève des enseignants en 1990, chaque événement compte ainsi ses pacificateurs du débat, se donnant pour mission épistolaire de prendre du recul et de proposer des solutions ${ }^{1}$.

Ainsi, ce lecteur-écrivant qui, en 1955, juge la guerre scolaire "puérile" : "

Il est à craindre que les réformes de l'enseignement ne viendront pas de si tôt, ce qui, pour notre pays, permettra à ces messieurs de la droite et de la gauche de se perfectionner encore dans le jeu de la petite guerre scolaire. (...) La guerre scolaire est puérile. Elle ne sert en fait qu'à «endoctriner» la jeunesse pour en faire de bons électeurs. Il serait bon pourtant d'apprendre aux jeunes comment penser et non ce qu'il faut penser ${ }^{2}$.

En 1961, un autre lecteur propose une solution pratique pour sortir de la grève contre la loi unique :

Il est nécessaire de modifier ce régime et le réadapter aux méthodes modernes, vu que depuis la guerre, les masses ont fortement évolué. (...) Comme l'intérêt général commande d'en finir avec cette grève, la solution pratique, sans nuire aux intérêts des parties, me paraît le vote de la loi unique, avec ajournement de son application jusqu'aux nouvelles élections ${ }^{3}$.

\footnotetext{
1 Nous ne pouvons évidemment rendre ici la diversité de ces nombreuses suggestions. Nous en relevons donc seulement quelques-unes, à titre représentatif.

2 E.H., dans Pourquoi Pas?, le 01/04/1955, n 1896 , p. 83-84

3 R.D., dans Pourquoi Pas?, le 13/01/1961, n 2198, p. 109.
} 
De même, en 1964, un lecteur écrit au Pourquoi Pas? pour inviter médecins et politiciens à dialoguer loyalement :

Je lis chaque semaine la rubrique de votre journal "On nous écrit", et je constate que beaucoup de lecteurs réclament, discutent et jugent selon une idée préconçue, ou sont insuffisamment informés sur l'objet de leur propos. (...) Que la loi soit mal faite, tout le monde l'admet. (...) Ici, chaque partie a tort et a raison. Dès le début (des " hostilités "), les médecins ont dit non! Et beaucoup d'entre eux, de leurs propres aveux, ne connaissaient pas le contenu de la loi. D'autre part, le gouvernement était engagé. Il s'est acharné. (...) Mais aucune des deux parties n'a commencé par se dire que d'une discussion loyale et sincère peut jaillir la lumière ${ }^{1}$.

Suite à la manifestation violente des agriculteurs à Bruxelles, plusieurs écrivants suggèrent des pistes pour "que cela ne se reproduise plus". Ainsi cette proposition adressée à la ville de Bruxelles pour mieux encadrer les manifestations à l'avenir : "Elle peut très bien poser certaines conditions, dont l'une devrait être l'existence de responsables payants, que ce soient les organisateurs eux-mêmes ou une assurance prise par ces derniers"2.

À l'instar de ces propos, en 1964, un lecteur "neutre" verra dans la crise de la sidérurgie l'expression d'un malaise sociétal profond. Il invitera dès lors les protagonistes à préférer un dialogue responsable au recours à la force :

Vous n'ignorez pas que cette même démocratie offre aux interlocuteurs sociaux tous les moyens de dialoguer (...) et cela autrement que par la violence ouverte. (...) En la circonstance, l'agressivité détournée a-t-elle vraiment pu aboutir à un résultat concret? N'est-ce pas plutôt le signe que quelque chose ne tourne plus rond au sein des appareils respectifs qu'ils soient gouvernementaux, patronaux ou syndicaux? (...) $\mathrm{Ne}$ serait-il pas temps d'effectuer une analyse austère, sincère et efficace des moyens et des matières précises qui permettent aux protagonistes de s'expliquer courageusement en hommes responsables et non en casseurs des rues $?^{3}$.

1 M.P., dans Pourquoi Pas ?, le 07/02/1964, n² 2358, pp. 141-142.

2 La Libre Belgique, le 18/04/1971, $\mathrm{n}^{\circ} 103, \mathrm{p} .2$.

3 LE HAEN, M, dans Le Soir, le 19/04/1982, n 90, p. 5. 
Outre leur volonté de pacification, certains écrivants "neutres" se plaisent aussi à relever les incohérences dans les discours des protagonistes. Tel ce lecteur qui s'interroge sur les divergences flagrantes entre les chiffres communiqués par la police et par les organisateurs lors d'une manifestation des fonctionnaires en juin 1986:

Au sujet de la manifestation socialiste du 31 mai, on a compté : 49.800 personnes selon la gendarmerie, (...) 200.000 personnes selon les dirigeants socialistes. (...) Il est inconcevable qu'en 1986 on puisse avoir des divergences de comptage pouvant aller de 1 à 4 , car il existe de nombreuses méthodes simples pour donner un ordre de grandeur valable et crédible pour réaliser de tels comptages ${ }^{\prime}$.

Et l'auteur du courrier de donner un exemple.

Enfin, en 1990, une lectrice résumera parfaitement l'enjeu du conflit de l'enseignement, tiraillé entre les perspectives à court et à long terme. Elle propose alors une solution provisoire pour enfin sortir de l'impasse :

Nous sommes coincés. L'éternelle alternative entre le court terme et le long terme se vit d'une manière cruciale et pénible dans ce problème d'enseignement : d'une part, à court terme, ces jeunes à la rue, à l'abandon, perturbés : d'autre part, à long terme, un avenir noir qu'il n'est pas possible d'accepter et une grève qui semble actuellement le seul moyen pour se faire entendre. (...) le temps n'est-il pas venu de trouver un compromis pratique (...) ? Par exemple, une trêve honnête, décidée de commun accord, limité dans le temps, qui permette de prendre du recul et de mettre en place certaines dispositions (...). Cette trêve se concrétiserait par une reprise en main des jeunes dans les écoles et des propositions claires de la part de nos dirigeants (...). Qu'on sorte enfin de ce dialogue de sourds !...2.

L'exclamation de cette lectrice traduit avec justesse tout l'enjeu polémique des grèves dont la rubrique du courrier des lecteurs se fait bien souvent l'écho.

1 L.Q., dans L'Echo de la Bourse, le 06-08/06/1986, n 108 , p. 14.

2 BEDORET, Ch., dans La Libre Belgique, le 10-11/11/1990, n 314-315, p. 2. 


\section{Conclusions}

Si ce que l'on classifie d'ordinaire de "presse d'opinion" a aujourd'hui quasiment disparu du paysage médiatique belge francophone, l'étude du courrier des lecteurs publié par cette même presse à l'occasion de grands conflits sociaux qui marquèrent l'aprèsguerre dans le pays tend à démontrer que, quelles que soient les époques, de grands courants de pensée ont par contre toujours animé l'opinion. Les journaux ont peut-être avec le temps perdu leur point de vue ou leur engagement politique. On ne peut en dire de même des lecteurs, pour qui la rubrique où est publié leur courrier n'a cessé de refléter l'expression d'opinions, souvent tranchées.

Au moins au travers de leur courrier de lecteurs, les journaux sont restés les vecteurs d'opinions, reflétant souvent l'arrière-pensée philosophique qui les sous-tend, mais ouvrant aussi largement le débat au sein de la société.

Les quelque 572 courriers parus à propos des divers conflits sociaux répertoriés dans cette attestent le réel désir du public de se positionner face aux mouvements en cours à l'époque. L'intérêt que la presse a manifesté à l'égard de ces prises de position n'a cependant pas été constant au cours de la période. Il semble patent que la prise en compte de l'expression du public des lecteurs, au moins à propos de questions sociales, croît avec le temps et se manifeste de manière beaucoup plus large en fin de période qu'au lendemain de la guerre.

Le nombre recensé de courriers publiés varie ainsi de 31 courriers dans les premières années pour atteindre 300 items lors de la grève des enseignants en 1990. Ce phénomène s'explique d'abord par le peu d'attention que la presse, hormis le Pourquoi Pas ?, a accordé au point de vue de ses lecteurs jusqu'au milieu des années 1970. Cet élément nous parâit significatif de l'évolution du rôle social de la presse dans la société et de la perception différente que la presse ellemême manifeste de ce rôle au fil du temps. Il faut toutefois ajouter que d'autres éléments peuvent également, en partie, expliquer cette évolution, comme la durée variable des faits concernés (d'un jour de manifestation à plusieurs mois de grèves ininterrompues) ou la teneur même des événements, certains touchant plus directement la population que d'autres et suscitant ainsi de plus vives réactions. 
Parmi les nombreux courriers publiés, de larges tendances se dégagent. D'abord, quant au profil des lecteurs-écrivants. Le grand public, et non les représentants des catégories socioprofessionnelles en grève, représente la majorité de ceux qui s'expriment dans les journaux. Faute de pouvoir (ou de vouloir) manifester de manière concrète leur mécontentement ou leur soutien, on peut considérer qu'ils prennent la plume pour faire partager leur avis et alimenter les débats au sein des journaux. La rubrique du courrier des lecteurs se fait dès lors essentiellement l'écho de ceux qui ne participent pas au mouvement, et s'avèrent fréquemment davantage critiques que favorables face à des événements qu'ils subissent et ne maîtrisent pas. Le courrier des lecteurs révèle ainsi une partie de la température sociale de la société, tout en restant évidemment dépendant des tendances qui affectent toujours le traitement médiatique, à savoir la tendance à l'hyperbole et à la schématisation.

L'étude a toutefois révélé que médecins et surtout enseignants faisaient exception à cette règle. Des membres de ces professions socialement privilégiées, impliqués dans des conflits sociaux les concernant, s'exprimeront assez largement dans la presse, se parant de revendications plus généreuses que les ouvriers, les agriculteurs ou les fonctionnaires. Médecins et enseignants disent ainsi se battre pour le bien de tous alors que les autres catégories professionnelles militent uniquement pour améliorer leur propre situation.

Par ailleurs, la nature même des mouvements sociaux donne matière à débat et fait donc de la rubrique du courrier des lecteurs un lieu essentiellement soumis aux controverses. Car face à une grève, on ne peut rester indifférent et l'opinion publique, telle qu'elle s'exprime dans le courrier des lecteurs, se divise spontanément entre ceux qui sont favorables au mouvement et ceux qui le condamnent. Qu'ils soient ou non grévistes, les lecteurs-écrivants se positionnent donc majoritairement en faveur ou en opposition vis-à-vis de la grève, attisant là les polémiques.

Cette tendance à l'expression d'opinions semble confirmée à la fois par la récurrence formelle des expressions utilisées, quel que soit le conflit social en cause, ainsi que par la permanence des thèmes abordés par les auteurs des lettres publiées. On relèvera ainsi que, à chaque occasion, les uns pointent du doigt les coupables, tentent de justifier leur action ou d'éveiller les consciences sur l'état de leur situation. Tandis que les autres, critiquant les instigateurs du 
mouvement, condamneront les méthodes "immorales" ou "violentes" des protestataires.

Entre ces deux pôles, l'étude a aussi relevé le positionnement de quelques lecteurs-écrivants "neutres", ne considérant pas la rubrique des lecteurs comme le lieu de la polémique mais celui de l'analyse et du diagnostic. Ceux-là souhaitent dépassionner le débat et proposent des solutions constructives pour sortir de la crise. Mais leur tâche n'est guère aisée dans un contexte si tranché. Au cœur de ces points de vue croisés, le journal se fait dès lors surtout le support des polémiques.

Face au surgissement de conflits sociaux ayant marqué la société, la rubrique des lecteurs a, de manière croissante au fil du temps, rempli un rôle de répercussions des opinions et attitudes de la population, ou plus exactement de la partie de la population souhaitant s'exprimer via les médias. A-t-elle contribué à développer réellement un débat au sein de la société, remplaçant ainsi les échanges verbaux de l'agora physique par le partage du dialogue social au sein d'une agora symbolique d'encre et de papier ? Le courrier publié lors de ces moments forts de la vie sociale belge semble davantage destiné à affirmer des points de vue qu'à engager le dialogue. Mais, comme nous le soulignions ci-dessus, il atteste surtout de la prégnance dans la population d'une volonté d'expression d'opinions. Une revendication que la presse semble avoir de mieux en mieux saisie au fil du temps, et qui l'a menée par la suite, à accorder de plus en plus de cas à la parole de ses usagers. 